Sinne des $\S 1$ HGB handelt und dieser bei Erwerb des Gemäldes gemäß § 344 HGB im Rahmen seines Handelsgeschäfts tätig wurde. Jedenfalls kann sich die Beklagte nicht auf die Verletzung der Rügepflicht berufen, weil sie den Mangel ihrerseits arglistig verschwieg, § 377 Abs. 5 HGB.

Nach eigenen Angaben wusste die Beklagte bzw. der für sie handelnde Vertreter bereits bei Angebotserstellung, dass das streitgegenständliche Werk nicht von dem 1881 verstorbenen JCB Püttner stammt. Auch lagen ihr keine Anhaltspunkte für die Existenz eines Künstlers mit denselben Initialen vor. Gleichwohl bot sie das Gemälde unter der Bezeichnung „JCB Püttner" an und musste dementsprechend damit rechnen, dass Kaufinteressenten dieses
Bild irrtümlich dem berühmten Maler zuschrieben, was dann auch unstreitig geschah. Allein das Fehlen von Literaturangaben und weiterer Hinweise auf den 1881 verstorbenen Künstler im Angebot genügte nicht, um die Verursachung eines Irrtums auf Käuferseite mit der erforderlichen Sicherheit ausschließen zu dürfen. Dies gilt umso mehr, als sich das Angebot der Beklagten nicht ausschließlich an spezialisierte Kunstkenner richtete, sondern an jedermann. Bereits aus diesem Grund hätte die Beklagte in ihr Angebot einen ausdrücklichen Hinweis aufnehmen müssen, dass das Gemälde nicht von dem 1881 verstorbenen Künstler stammt. Dieses Unterlassen begründet den Arglistvorwurf.

$[\ldots]$

\title{
Kunstfreiheit versus Tierschutz
}

Eine Rechtsordnung, die das im Rahmen einer Performance inszenierte Töten eines Wirbeltiers akzeptiert, kann von den Menschen nicht verlangen, dass diese sich gegenüber Tieren verantwortungsvoll verhalten. Es ist ein Unterschied, ob ein Nutztier getötet wird, um es seiner Bestimmung zuzuführen oder ob ein solches Tier vor einem Publikum getötet wird, um durch den Akt des Tötens zu beeindrucken. (Leitsatz d. Redaktion)

$[\ldots]$

Der Angeklagte R. wird wegen Tötens eines Wirbeltiers ohne vernünftigen Grund zu einer Geldstrafe von achtzig Tagessätzen zu je 30 Euro verurteilt. Der Angeklagte N. wird wegen Tötens eines Wirbeltiers ohne vernünftigen Grund zu einer Geldstrafe von fünfzig Tagessätzen zu je 20 Euro verurteilt. Die Angeklagte B. wird wegen Tötens eines Wirbeltiers ohne vernünftigen Grund in Tateinheit mit Zufügen erheblicher Leiden bei einem Wirbeltier aus Rohheit zu einer Geldstrafe von zwanzig Tagessätzen zu je 30 Euro verurteilt.

Die Angeklagten haben die Kosten des Verfahrens und ihre notwendigen Auslagen zu tragen.

R.: §§ 17 Nr. 1 TierSchG, 25 Abs. 2 StGB

N.: $\S § 17$ Nr. 1 TierSchG, 25 Abs. 2 StGB

B.: §§ $17 \mathrm{Nr} .1$ und Nr. 2 a TierSchG, 25 Abs. 2 StGB
Gründe:

(abgekürzte Fassung gemäß § 267 Abs. 4 StPO bezüglich der Angeklagten B.)

I.

1. Der Angeklagte R. ist 44 Jahre alt, ledig und deutscher Staatsangehöriger. Er ist in bürgerlichen Verhältnissen in einer niedersächsischen Kleinstadt aufgewachsen und hat die schulische Ausbildung mit dem Abitur abgeschlossen. Er leistete den Zivildienst $a b$ und begann in Berlin ein Kunststudium, das er vorzeitig abbrach. Anschließend übte der Angeklagte verschiedene Hilfstätigkeiten aus, u.a. als Beleuchter bei Dreharbeiten des ZDF. Seit mehreren Jahren ist der Angeklagte beruflich als selbstständiger, bildender Künstler tätig. Der Angeklagte hat aus drei verschiedenen Beziehungen Kinder im Alter von 8, 11 und 16 Jahren. Er hat nach eigenen Angaben als Künstler ein Jahresnettoeinkommen von 4.000,00 €. Der Angeklagte ist nicht vorbestraft. 
2. Der Angeklagte N. ist 55 Jahre alt, ledig und deutscher Staatsangehöriger. Er ist in Bayern geboren und zog mit 18 Jahren nach Berlin, um hier Design zu studieren. 1977 eröffnete der Angeklagte, der sich selbst als Künstler bezeichnet, eine so genannte Bio-Metzgerei in Berlin-Schöneberg. Der kinderlose Angeklagte ist arbeitslos und bezieht Arbeitslosengeld II; einschließlich seiner Einkünfte aus einer geringfügigen Beschäftigung hat der Angeklagte ein monatliches Nettoeinkommen von 600,00 €. Der Angeklagte ist vorbestraft. Sein Registerauszug vom 17. April 2007 enthält insgesamt 8 Eintragungen. Mit Urteilen vom 25. Juli 1990 und vom 25. Oktober 1991 verurteilte das Amtsgericht Berlin-Tiergarten den Angeklagten, dessen Bio-Metzgerei nach eigenen Angaben mehrfach - u.a. von der Fachzeitschrift „Der Feinschmecker" - ausgezeichnet wurde, wegen Verstößen gegen das Lebensmittel- und Bedarfsgegenständegesetz zu Geldstrafen. Es folgten Verurteilungen zu Geldstrafen wegen Verkehrsdelikten, einem Verstoß gegen das Betäubungsmittelgesetz, wegen Einkommenssteuerhinterziehung sowie zuletzt mit Urteil vom 3. November 2004 wegen Diebstahls.

3. Die Angeklagte B. ist im ehemaligen Karl-MarxStadt geboren. Sie ist deutsche Staatsangehörige und 29 Jahre alt. Die Angeklagte ist ledig und kinderlos. Als Friseurin hat sie ein monatliches Nettoeinkommen von 900,00 €. Die Angeklagte ist nicht vorbestraft.

\section{II.}

Am 17. Februar 2006 gegen 22.00 Uhr, in der Galerie "Monsterkeller", Rosenthaler Straße 39, 10178 Berlin inszenierte der Angeklagte R. eine Vorführung. Wie von den Angeklagten zuvor vereinbart, hielt der Angeklagte N. zwei zuvor zu diesem Zweck erworbene Kaninchen fest, während die Angeklagte B. den Tieren durch Drehen der Köpfe jeweils das Genick brach. Der Angeklagte hatte die Vorführung als Kunstaktion konzipiert. Er wollte „scheinbar akzeptierte kulturelle Rituale abbilden, um die überhöhte spirituelle Reflektion des westlichen Kulturmenschen durch Diskussion dieser kontroversen Themen zu hinterfragen". Die Kaninchen sollten sieben Tage später verspeist werden. Ob dies tatsächlich geschah, hat in der Hauptverhandlung nicht geklärt werden können. Der Angeklagte R. hatte der Angeklagten B. versichert, die Vorführung vom 17. Februar 2002 sei legal, angemeldet und von der Kunstfreiheit gedeckt.

\section{III.}

Die Feststellungen beruhen auf den glaubhaften Einlassungen der Angeklagten, ihren ebenfalls glaubhaften Angaben zu ihren persönlichen Verhältnissen und den in der Hauptverhandlung verlesenen Registerauszügen.

IV.

Die Angeklagten haben sich wie tenoriert schuldig gemacht.

1. Die Angeklagten R. und N. sind des Vergehens des Tötens eines Wirbeltiers ohne vernünftigen Grund strafbar (§ 17 Nr. 1 TierSchG); hinsichtlich eines Verstoßes gegen $\S 17 \mathrm{Nr}$. 2 a TierSchG wurde die Strafverfolgung mit Zustimmung der Staatsanwaltschaft gem. § 154a Abs. 2 StPO beschränkt. Die Angeklagten handelten im bewussten und gewollten Zusammenwirken aufgrund eines gemeinschaftlich gefassten Tatentschlusses und damit als Mittäter im Sinne von $\S 25$ Abs. 2 StGB.

Die Angeklagten können sich nicht erfolgreich auf die Grundrechte der Kunst-, Meinungsfreiheit oder Berufsfreiheit berufen (a); auch aus dem Umstand, dass die Kaninchen eine Woche nach der Tat verspeist werden sollten, ergibt sich nichts Abweichendes (b)

a) Einer Verurteilung der Angeklagten stehen weder die Grundrechte der Kunstfreiheit, Art. 5 Abs. 3 GG, noch die der Meinungsäußerungs- oder Berufsfreiheit, Art. 5 Abs. 1 und Art. 12 Abs. 1 GG, entgegen. Dabei kann dahinstehen, welchem Kunstbegriff das Gericht folgt und ob das Verhalten der Angeklagten nach den verschiedenen Kunstbegriffen in den Schutzbereich der Kunstfreiheit fallen würde. Ohne Belang ist auch, ob Art. 5 Abs. 3 GG einen selbstständigen Rechtfertigungsgrund darstellt oder ob die Kunstfreiheit bei der Auslegung des § 17 Nr. 1 TierSchG und des Begriffs „vernünftiger Grund“ zu berücksichtigen wäre.

aa) Denn auch wenn die Vorführung einschließlich des Tötens der Kaninchen in den Schutzbereich des Art. 5 Abs. 3 GG fallen sollte, bedeutet dies nicht, dass die Inszenierung außerhalb des Strafrechts steht. Das Grundgesetz hat die Kunstfreiheit zwar nicht unter einen Gesetzesvorbehalt gestellt. Schranken der Kunstfreiheit können sich daher nur aus dem Grundgesetz selbst ergeben, nämlich dann, wenn Grundrechte anderer Personen oder sonstige Güter mit Verfassungsrang beeinträchtigt sind (Jarass/Pieroth, Kommentar zum Grundgesetz, 9. Auflage, 2007, Art. 
$5 \mathrm{Rn} 113)$. Es ist in diesem Fall zwischen den kollidierenden Rechtsgütern abzuwägen und festzustellen, welches Rechtsgut im Einzelfall Vorrang erhalten und welches hinter dem vorrangigen Recht zurücktreten muss (Beschluss des Bundesverfassungsgerichts vom 1.3.1978, 1 BvR 333/75, BVerfGE 47, 327; Urteil des Bundesverfassungsgerichts vom 24.4.1985, 2 BvF 2, 3, 4/83, BVerfGE, 69, 1).

bb) Gemessen an diesen Grundsätzen könnten die Angeklagten sich nicht auf das Recht der Kunstfreiheit berufen. Denn bei dem Tierschutz handelt es sich um ein Gut mit Verfassungsrang (1), das hier der Kunstfreiheit vorginge (2).

(1) Durch Gesetz zur Änderung des Grundgesetzes vom 26. Juli 2002 ist das Grundgesetz in Art. 20a GG um das Staatsziel „Tierschutz" ergänzt worden. Ausweislich der amtlichen Begründung zu dem Gesetzesentwurf (BT-Drucks. 14/8860 S. 1, 3) sollte mit der Aufnahme des Staatsziels "Tierschutz" dem "Gebot eines sittlich verantworteten Umgangs des Menschen mit Tieren Rechnung getragen werden". Die Ergänzung des Grundgesetzes beruhte nach dem - fraktionsübergreifend eingebrachten - Gesetzesentwurf auf der Annahme, dass „der Umgang insbesondere mit höher entwickelten Tieren ein ethisches Mindestmaß für das menschliche Verhalten erfordere". Als Folge der Aufnahme des Tierschutzgedankens in Art. 20a GG ist das Staatsziel "Tierschutz" gegenüber anderen Verfassungsnormen einschließlich Grundrechten grundsätzlich gleichrangig (Murswiek in: Sachs, Kommentar zum Grundgesetz, 3. Auflage, 2002, Art 20 a Rn. 55).

Mit dem Vorstellungen des Gesetzgebers über einen verantwortungsvollen Umgang mit Tieren ist das Verhalten der Angeklagten nicht vereinbar. Gegenstand der Vorführung war das Töten der Kaninchen. Der Akt des Tötens war dabei nicht dem späteren Verzehr untergeordnet, sondern stand im Vordergrund. Der Angeklagte R. wollte durch das inszenierte Töten bei den Zuschauern Gefühle der Betroffenheit - mag es sich um Abscheu, Angst, Mitleid handeln - hervorrufen. Einen sittlich verantworteten Umgang stellt ein Verhalten nicht dar, bei dem ein eigenständiges Lebewesen getötet wird, um durch den Tötungsakt das Publikum zu beeindrucken. Das Gericht hält auch den Einwand, die Kaninchen seien ohnehin dafür bestimmt gewesen, getötet und verzehrt zu werden, nicht für erheblich. Es ist ein Unterschied, ob ein Nutztier getötet, um es seiner Bestimmung zuzuführen oder ob ein solches Tier vor einem Publikum getötet wird, um durch den Akt des Tötens zu beeindrucken. Im Übrigen darf die mögliche Außenwirkung der Vorführung nicht übersehen werden. Eine Rechtsordnung, die das im Rahmen einer Performance inszenierte Töten eines Wirbeltiers akzeptiert, kann von den Menschen nicht verlangen, dass diese sich gegenüber Tieren verantwortungsvoll verhalten. Genau dies war aber das Anliegen der Ergänzung des Art. 20a GG.

(2) Bei der Abwägung der konkurrierenden Verfassungsgüter - Kunstfreiheit gegen Staatsziel Tierschutz - hätte der Tierschutz hier Vorrang. Dem Angeklagten R. ist zwar darin zuzustimmen, dass „Kunst nicht allein ein schönes Stück Öl als Blickfang und Dekoration für das Wohnzimmer oder Büro beinhaltet". Ebenso wenig reduziert sich Kunst aber auf den inszenierten Tötungsakt von Wirbeltieren. Soweit es dem Angeklagten R. darum ging „, die überhöhte spirituelle Reflektion des westlichen Kulturmenschen zu hinterfragen", so lässt ihm die Rechtsordnung unzählige Wege frei, dieses Ansinnen zu erreichen.

Aus entsprechenden Gründen können sich die Angeklagten nicht auf das Grundrecht der Meinungsfreiheit, Art. 5 Abs. 1 GG, oder der Berufsfreiheit, Art. $12 \mathrm{GG}$ berufen.

b) Der Strafbarkeit der Angeklagten steht auch nicht entgegen, dass der Angeklagte R. beabsichtigte, die Kaninchen eine Woche nach der Vorführung zu verspeisen. Grundsätzlich stellt es zwar einen vernünftigen Grund im Sinne des § 17 Nr. 1 TierSchG dar, wenn ein Wirbeltier zum Zwecke des Verzehrs getötet wird (Lorz/Albert, Kommentar zum Tierschutzgesetz, 5. Auflage, 1999, § 17 Rn. 19). Dies gilt indes nur, wenn die konkrete Handlung der Fleischgewinnung dient (Lorz/Albert, aaO). Hier diente das Töten der Kaninchen nicht der Fleischgewinnung, sondern war Gegenstand der Inszenierung des Angeklagten R. Es ging bei dem Töten der Tiere nicht darum, Fleisch zu gewinnen; sondern der Angeklagte R. wollte die Zuschauer durch den Akt des Tötens der Kaninchen beeindrucken.

2. Nachdem die Angeklagte B. den gegen den Strafbefehl vom 12. Februar 2007 eingelegten Einspruch auf die Rechtsfolgen beschränkte, ist der Schuldspruch aus dem Strafbefehl in Rechtskraft erwachsen; die Angeklagte hat sich entsprechend nach den §§ 17 Nr. 1 und Nr. 2a TierSchG strafbar gemacht.

\section{V.}

Das Gericht hat bei einem Strafrahmen von Geldstrafe bis zu drei Jahren Freiheitsstrafe jeweils auf Geldstrafen von 80 Tagessätzen - für den Angeklagten R. -, 50 Tagessätzen - für den Angeklagten 
N. - und 20 Tagessätzen - für die Angeklagte B. erkannt.

1. Bei der Strafzumessung hinsichtlich des Angeklagten R. war zu seinen Gunsten seine straffreie Führung zu berücksichtigen; strafmildernd hat das Gericht außerdem berücksichtigt, dass er seinen führenden - Tatbeitrag freimütig eingeräumt hat. Auch der Umstand, dass der Angeklagte glaubte, im höheren Interesse der Kunst zu handeln, war zu seinen Gunsten zu werten. Strafschärfend fiel dagegen ins Gewicht, dass der Angeklagte eine Form des Tötens gewählt hatte, die aus Sicht des Betrachters besonders grausam war. Dies ist dies bei der Strafzumessung des $\S 17 \mathrm{Nr}$. 1 TierSchG zu berücksichtigen. Denn bei einer grausamen Art einer inszenierten Tötung besteht das Risiko, dass die Zuschauer in besonderem Maße verrohen.

Die Tagessatzhöhe hat das Gericht mit 30 Euro bestimmt. Dabei ist es davon ausgegangen, dass der gesunde und nicht unintelligente Angeklagte, der nach eigenen Angaben ein Jahresnettoeinkommen von lediglich 4.000,00 € hat, ohne Schwierigkeiten einer Tätigkeit nachgehen könnte, bei derer ein Nettoeinkommen von monatlich $900.00 €$ erzielen könnte.

2. Für den Angeklagten N. hat das Gericht auf eine tat- und schuldangemessene Geldstrafe von
50 Tagessätzen erkannt. Zu seinen Gunsten hat das Gericht den geringen Tatbeitrag berücksichtigt. Strafschärfend fielen die Vorstrafen ins Gewicht. Die Tagessatzhöhe hat das Gericht nach den persönlichen und wirtschaftlichen Verhältnissen des Angeklagten mit $20 €$ bestimmt ( $\$ 40$ Abs. 2 StGB).

3. Gegen die Angeklagte B. war eine Geldstrafe von 20 Tagessätzen erforderlich aber auch ausreichend. Strafschärfend fiel ihr maßgeblicher Tatbeitrag bei der unmittelbaren Tatausführung ins Gewicht. Zu Gunsten der Angeklagten war zu berücksichtigen, dass diese bisher nicht vor Gericht stand. Im Übrigen ist das Gericht zu Gunsten der Angeklagten davon ausgegangen, dass diese die Tragweite ihres Handelns - der Angeklagte R. hatte ihr versichert, die Handlungen seien legal und angemeldet - nicht überblickte. Im Gegensatz zu dem Angeklagten N., der zu der Verhandlung mit einer Schirmmütze erschien, auf der eine Website mit dem Namen „www. kunstkaninchen.de" gedruckt war, machte die Angeklagte im Übrigen einen einsichtigen Eindruck. Anders als die Mitangeklagten bereute die Angeklagte B. die Tat. Die Tagessatzhöhe hat das Gericht nach den persönlichen und wirtschaftlichen Verhältnissen der Angeklagten mit $30 €$ bestimmt ( $\$ 40$ Abs. $2 \mathrm{StGB})$.

[...]

\section{Literatur}

\section{Kulturgüterschutz - internationale und nationale Aspekte}

Gilbert H. Gornig / Hans-Delef Horn / Dietrich Murswiek (Hrsg.)

- Der Schutz von Kulturgütern hat viele Facetten und wirft ebenso viele Fragen auf. Mit ausgewählten rechtlichen Aspekten des Kulturgüterschutzes hat sich die 23. Staats- und Völkerrechtliche Fachtagung der Studiengruppe für Politik und Völkerrecht im November 2005 in Stuttgart-Hohenheim befasst. Im Mittelpunkt stand der rechtliche Rahmen für den Schutz von Kulturgütern in und ihre Rückführung nach einem Krieg, mithin eine ebenso spezielle wie zugleich auch komplexe Komponente des Kulturgüterschutzes. Die auf diesem Symposium gehaltenen Vorträge sind nun von den Veranstaltern als Tagungsband herausgegeben worden.

Die einzelnen Beiträge sind höchst unterschiedlichen Charakters und unterschiedlicher Qualität. Zwei Abhandlungen verdienen besondere Beach- tung: In seinem einführenden Referat gibt Gilbert H. Gornig einen verlässlichen Überblick über die wesentlichen Prinzipien, Begriffe und Rechtsquellen des internationalen Kulturgüterschutzes. Dabei wird deutlich, dass das Kulturgüterschutzrecht zum Teil noch an erheblichen Defiziten und Diskrepanzen leidet. Es fehlt an verbindlichen und handhabbaren Begriffsdefinitionen, angefangen etwa beim Gegenstand dieses Rechtsgebiets, dem Kulturgut, und mangelt mitunter an Kompatibilitäten zwischen den verschiedenen Regelungen, die von der lokalen über die regionale und nationale bis hin zur supranationalen und internationalen Ebene zum rechtlichen Kulturgüterschutz getroffen wurden und werden. Dieser Befund muss freilich nicht schlicht als entsprechender Normierungsbzw. Harmonisierungswunsch verstanden werden,
Duncker \& Humblot Berlin 2007, 272 S. 78,- Euro ISBN:

978-3-428-12525-8 